ZAMM · Z. Angew. Math. Mech. 78 (1998) 4, 243-253

Bhattacharyya, S.; Pal, A.; Datta, N.; Nath, G.

\title{
Unsteady Rotating Flow of a Compressible Fluid over a Finite Disk
}

\begin{abstract}
The semi-similar formulation of the problem of an unsteady boundary layer over a stationary disk of finite radius induced by a time dependent rotating flow of compressible fluid is considered. The free stream swirling flow is assumed to be tangential and varies as a power of radial distance, i.e., $v_{e} \sim r^{-n} \varphi\left(t^{*}\right)$, where $\varphi\left(t^{*}\right)$ is an arbitrary function of time $t^{*}$. Several free stream velocity distributions have been examined. Near the outer edge of the disk the flow is described by the Stewartson edge similarity solutions and near the axis of rotation the solutions coincide with the Bödewadt's type of similarity solution. The velocity profiles are monotonic over the outer half of the finite disk but they exhibit oscillation over the inner half (for $n<1$ ). Due to reversal in radial velocity over the inner half of the disk, the boundary layer equations become a kind of time dependent singular parabolic equation and the solution of which requires the conditions over all of its boundaries. Finite-difference scheme using space-centered differences in the axial direction and upwind differences in the radial direction is used for solving the governing equations. Crank-Nicolson scheme is used for timewise discretisation. Numerical solutions have been obtained for the entire range of the radial distance. Solutions for the entire range of the radial distance exist upto a certain critical value of n. Boundary layer solutions do not exit over the inner half of the disk for the case of potential vortex flow $(n=1)$. Effect of acceleration and oscillation of the free stream azimuthal velocity on the boundary layer flow and heat transfer have been investigated.
\end{abstract}

MSC (1991): 76N20, 76U05

\section{Introduction}

Studies of flow near rotating disks are a recurring theme in the fluid mechanics literature. The most fundamental of these, namely that of the flow above a single, infinite rotating disc, was treated originally by VON KARMAN [1]. Due to the infinite domain of the problem, the radial and azimuthal velocity components can be assumed to have a simple, linear dependence upon radius. The axial velocity and pressure are independent of radius, dependent solely upon the axial distance from the disk. For these the Navier-Stokes equations could be reduced, in this case, to a set of ordinary differential equations, using a similarity transformation. The boundary layer development of steady incompressible axisymmetric rotating flow over a stationary disk of finite radius has been studied by several authors ([1]-[8]). It was found that the boundary layer over the finite disk spreads inwards from the outer edge and it admits of a similarity solution of Stewartson type at the edge of the disk and a similarity solution of Bödewadt's type near the center of the disk. The velocity profiles near the edge of the disk are found to be non-oscillatory but over the inner half of the disk the velocity profiles exhibit oscillations about their free stream values.

The disturbance to the boundary layer solutions caused by the finite size of the disk originates at the outer edge $(r=1)$. Then it travels inwards at a speed equal to the negative radial component of the fluid velocity and simultaneously diffuses instantaneously in the axial direction. BELCHER et al. [4] have shown that if the circulation of the outer flow is an increasing function of the radial distance then the radial velocity of the boundary layer can change sign for $r<1$. In the flow reversal regions, numerical integration of the boundary layer equations becomes complicated. BELCHER et al. [4] overcame this difficulty by introducing time as an independent variable and carried out the integration forward in time until the solution is substantially stable. VAN DE VOOREN et al. [6] have solved the boundary value problem describing the motion by a line iteration and under-relaxation in the same way for an elliptic problem.

The compressibility effect on boundary layer over a disk of finite radius has been considered by TOREN and SolAN $[9,10]$. They have applied a modified momentum integral technique to investigate the flow with an isothermal assumption and subsequently obtained a similarity solution which is valid at the edge of the disk.

The class of rotating flows in which the external tangential velocity $v_{e}$ varies as a power of the distance $r$ from the axis of rotation is termed as a generalised vortex. The form of the velocity $v_{e} \sim r^{-n}$, has been observed to be the best fit in explaining certain types of atmospheric vortex flow or motion in a vortex chamber. For example, the value of $n$ between 0.4 and 0.6 is appropriate to hurricanes. The principle interest here is the range $-1 \leq n \leq 1$. KING and LEWELLEN [11] have studied the generalised vortex motion of an incompressible fluid set up over a stationary infinite disk. They encountered certain difficulties in solving the boundary layer similarity equations for $n>0.1$. BURGGRAF et al. [12] and BELCHER et al. [4] examined the solution of the flow due to a generalised vortex set up over a finite disk and found that the boundary layer takes a double structure for $n=1$ (potential vortex) and a multiple or ladder structure for $0.1217<n<1.0$.

In the present work, we have studied the unsteady generalised vortex flow of a compressible fluid set up over a stationary finite disk kept at a uniform temperature. We have considered a continuous time dependent variation of the free stream azimuthal velocity. The unsteady boundary layer equations have been solved through a forward marching in time with the corresponding steady-state solution as the initial condition. The boundary conditions are governed by the edge similarity solutions at $r=1$ (edge of the disk) and the terminal similarity solutions near the center. Using the 
condition at $r=1$ numerical solutions have been obtained by forward integration from the edge of the disk inward till the radial flow in the boundary layer remains inward everywhere. But over the inner half of the disk, radial flow reversal (outward) takes place (for $n<0$ ) which changes the character of the boundary layer equations and the boundary layer solutions depend also on the condition at $r=0$. A second-order accurate upwind difference scheme has been employed for discretisation of terms involving derivatives with respect to $r$. It has been proved that the terminal similarity solution (at $r=0)$ does not exist for potential vortex flow $(n=1)$.

The response of the boundary layer due to time-dependent variation of the free stream has many practical applications. Specifically, it is of interest in missile aerodynamics, turbomechanics, unsteady nozzle flow etc. The fluctuating free stream boundary layers and the allied unsteady flows have been extensively reviewed by TELIONIS [13].

\section{Formulation}

We consider a cylindrical polar co-ordinate system $(r, \theta, z)$ with $r$ measuring distance from $z$-axis. An infinite volume of fluid occupies all of the space except for a fixed finite disk defined by $z=0,0<r<a$. The velocity of the fluid at almost all points of space, but specifically not in the neighbourhood of the plane, is circulatory with the azimuthal velocity $v_{e}=v_{0}(r / a)^{-n} \varphi\left(t^{*}\right)$, where $v_{0}$ is the velocity of the free stream at a particular radial distance $a$, the parameter $n$ determines the nature of vortex flow, and $\varphi\left(t^{*}\right)$ is a continuous function of the non-dimensional time $t^{*}$ which measures the unsteadiness of the free stream. The principal interest of $n$ is between -1 (rigid-body rotation) and 1 (potential vortex)

In the governing unsteady, compressible boundary layer equations in cylindrical polar co-ordinates (Ref. RILEY [15]) we introduce a co-ordinate $Z$ and a stream function $\psi$ such that

$$
Z=\int_{0}^{z}\left(\varrho / \varrho_{e}\right) \mathrm{d} z, \quad u=r^{-1} \frac{\partial \psi}{\partial Z}
$$

and the following transformations for $0<r<a$ (Ref. BELCHER et al. [4]):

$$
\begin{aligned}
t^{*} & =\left(v_{0} / a\right)(1-\xi)^{-(n+1)} t, \quad \xi=1-r / a \\
\eta & =(Z / a)\left(v_{0} a / v_{e}\right)^{1 / 2}(1-\xi)^{-(n+1) / 2} \xi^{-1 / 4}, \\
\psi & =v_{0} a^{2}\left(v_{0} a / v_{e}\right)^{-1 / 2}(1-\xi)^{-(n-3) / 2} \xi^{3 / 4} f\left(t^{*}, \eta, \xi\right) \varphi\left(t^{*}\right), \\
v & =v_{0}(1-\xi)^{-n} g\left(t^{*}, \eta, \xi\right) \varphi\left(t^{*}\right), \quad T=T_{e} \theta\left(t^{*}, \eta, \xi\right), \quad F=f_{\eta} .
\end{aligned}
$$

Consequently the governing boundary layer equations are reduced to the following non-dimensional form:

$$
\begin{array}{lr}
\left(N F_{\eta}\right)_{\eta}+n \varphi \xi F^{2}+(1 / 2) \varphi(1-\xi) F^{2}-(3 / 4) \varphi(1-\xi) f F_{\eta}-\varphi \xi(1-\xi) f_{\xi} F_{\eta} & \\
-((n-3) / 2) \varphi \xi f F_{\eta}+g^{2} \varphi-\theta \varphi-\xi^{1 / 2} F_{t^{*}}-\xi^{1 / 2} F \varphi_{t^{*}} \varphi^{-1} & =-\varphi \xi(1-\xi) F F_{\xi}, \\
\left(N g_{\eta}\right)_{\eta}+(n-1) \varphi \xi g F-n((n-3) / 2) \varphi \xi f g_{\eta}-(3 / 4) \varphi(1-\xi) f g_{\eta}-\varphi \xi(1-\xi) g_{\eta} f_{\xi} & =-\varphi \xi(1-\xi) F g_{\xi}, \\
-\xi^{1 / 2} g_{t^{*}}-\xi^{1 / 2} g \varphi_{t^{*}} \varphi^{-1}+\xi^{1 / 2} \theta \varphi_{t^{*}} \varphi^{-1} & =-\varphi \xi(1-\xi) F \theta_{\xi} . \\
(1 / \operatorname{Pr})\left(N \theta_{\eta}\right)_{\eta}+E \varphi F \theta \xi+N E \xi F_{\eta}^{2}+N E g_{\eta}^{2}-((n-3) / 2) \varphi \xi f \theta_{\eta}-(3 / 4) \varphi(1-\xi) f \theta_{\eta} \\
-\varphi \xi(1-\xi) f_{\xi} \theta_{\eta}-\xi^{1 / 2} \theta_{t^{*}}
\end{array}
$$

The boundary conditions are given by

$$
\begin{aligned}
& \eta=0: \quad f=0, \quad F=0, \quad g=0, \quad \theta=\theta_{w}, \\
& \eta \rightarrow \infty: \quad F=0, \quad g=1, \quad \theta=1 \text {. }
\end{aligned}
$$

Here $u, v$, and $w$ are the radial, tangential, and axial components of velocity, respectively. The temperature is denoted by $T$. The functions $f, F$, and $g$ are functions of the non-dimensional variables $\eta, t^{*}$, and $\xi$ and they represent respectively the dimensionless axial, radial, and tangential velocity components. The non-dimensional function $\theta\left(t^{*}, \eta, \xi\right)$ represents the temperature distribution in the boundary layer. The density is denoted by $\varrho$, viscosity by $\mu$, specific heat at a constant pressure by $C_{p}$, and the kinematic viscosity by $\nu$. Subscripts $\eta, t^{*}$, and $\xi$ denote respectively the partial derivatives with respect to the corresponding variable. Here the suffixes $e$ or $w$ are used to denote that the quantity has been measured at infinity or at the wall, respectively.

A power law relation between viscosity and temperature has been assumed: $\mu \propto T^{\omega}$, where $0.5 \leq \omega \leq 1$.

Consequently, $N=\varrho \mu / \varrho_{e} \mu_{e}=\theta^{\omega-1}$.

The Prandtl number $\operatorname{Pr}=\mu C_{p} / k, k$ is the thermal conductivity. $E=v_{e}^{2} / C_{p} T_{e}$ is the dissipation function and $M=v_{0} / c$ is the Mach number. $E_{1}=(\gamma-1) M^{2}$ is the dissipation parameter, $c$ is the velocity of sound, and $\gamma$ is the ratio of specific heats which has been taken as 1.4 for air. So,

$$
E=E_{1}(1-\xi)^{-2 n}, \quad 0<\xi<1 .
$$


It may be noted that the equations (2)-(4) can be reduced to the set of equations considered by BELCHER et al. [4] for the case of incompressible fluid $(\theta=1)$.

Following [5] it may be noted that the flow near the edge of the disk $(\xi=0)$ is resembled to the STEWARTSON similarity solution [2]. Again the flow near the center $(\xi=1)$ is independent of the edge effect and is similar to the BÖDEWADT's similarity solution [14]. Hence the two conditions in $\xi$ are governed by the solution of two sets of ordinary differential equations which can be obtained as

$$
\begin{aligned}
& \left(N F_{\eta}\right)_{\eta}+(1 / 2) \varphi F^{2}-(3 / 4) \varphi f F_{\eta}+g^{2} \varphi-\theta \varphi=0 \\
& \left(N g_{\eta}\right)_{\eta}-(3 / 4) \varphi f g_{\eta}=0 \\
& (1 / \operatorname{Pr})\left(N \theta_{\eta}\right)_{\eta}+N E_{1} g_{\eta}^{2}-(3 / 4) \varphi f \theta_{\eta}=0
\end{aligned}
$$

when $\xi=0$,

$$
\begin{aligned}
& \left(N F_{\eta}\right)_{\eta}+n \varphi F^{2}-((n-3) / 2) \varphi f F_{\eta}+g^{2} \varphi-\theta \varphi-F_{t^{*}}-F \varphi_{t^{*}} \varphi^{-1}=0 \\
& \left(N g_{\eta}\right)_{\eta}+(n-1) \varphi g F-((n-3) / 2) \varphi f g_{\eta}-g_{t^{*}}-g \varphi_{t^{*}} \varphi^{-1}+\theta \varphi_{t^{*}} \varphi^{-1}=0 \\
& (1 / \operatorname{Pr})\left(N \theta_{\eta}\right)_{\eta}-((n-3) / 2) \varphi f \theta_{\eta}-\theta_{t^{*}}=0
\end{aligned}
$$

$$
\text { when } \xi=1 \text {. }
$$

In accordance with BELCHER et al. [4] we considered that the steady-state solution is established immediately at the edge of the disk.

The boundary conditions for both sets of equations (6) and (7) are given by (5). Moreover, both the conditions over $\xi$ are consistent with the equations (2)-(4) as these satisfy equations (2)-(4) for $\xi=0$ and $\xi=1$.

The set of equations $(6)$ governing the flow at the edge $(\xi=0)$ is a set of ordinary differential equations which indicates that the steady-state solution is established immediately at the edge of the disk [4]. ToREN and SolAN [10] have studied the edge similarity equation and the set of unsteady equations $(7 \mathrm{a})-(7 \mathrm{~b})$ valid near $\xi=1$ were studied by BHATTACHARYYA et al. [16].

The initial conditions $\left(t^{*}=0\right)$ for the set of equations $(2)-(4)$ are governed by the solution of the corresponding steady-state equations, which can be obtained by setting in equations $(2)-(4)$, the following relations:

$$
\varphi(0)=1, \quad \varphi_{t^{*}}=F_{t^{*}}=g_{t^{*}}=\theta_{t^{*}}=0 .
$$

The steady-state equations are expressed as

$$
\begin{array}{lr}
\left(N F_{\eta}\right)_{\eta}+n \xi F^{2}+(1 / 2)(1-\xi) F^{2}-(3 / 4)(1-\xi) f F_{\eta}-\xi(1-\xi) f_{\xi} F_{\eta} & \\
-((n-3) / 2) \xi f F_{\eta}+g^{2}-\theta & =-\xi(1-\xi) F F_{\xi}, \\
\left(N g_{\eta}\right)_{\eta}+(n-1) \xi g F-((n-3) / 2) \xi f g_{\eta}-(3 / 4)(1-\xi) f g_{\eta}-\xi(1-\xi) g_{\eta} f_{\xi}=-\xi(1-\xi) F g_{\xi} \\
(1 / \operatorname{Pr})\left(N \theta_{\eta}\right)_{\eta}+E F \theta \xi+N E \xi F_{\eta}^{2}+N E g_{\eta}^{2}-((n-3) / 2) \xi f \theta_{\eta} & =-\xi(1-\xi) F \theta_{\xi} . \\
-(3 / 4)(1-\xi) f \theta_{\eta}-\xi(1-\xi) f_{\xi} \theta_{\eta}
\end{array}
$$

The initial conditions for the set of equations (7) are governed by the following ordinary differential equations:

$$
\begin{aligned}
& \left(N F_{\eta}\right)_{\eta}+n F^{2}-((n-3) / 2) f F_{\eta}+g^{2}-\theta=0, \\
& \left(N g_{\eta}\right)_{\eta}+(n-1) g F-((n-3) / 2) f g_{\eta}=0, \\
& (1 / \operatorname{Pr})\left(N \theta_{\eta}\right)_{\eta}-((n-3) / 2) f \theta_{\eta}=0 .
\end{aligned}
$$

\section{Numerical method}

The set of partial differential equations (2)-(4) are bi-parabolic, namely having two parabolic directions $t^{*}$ and $\xi$. We have used Crank-Nicolson scheme for discretising the time derivatives in equations (2)-(4). The steady-state solutions, i.e., at $t^{*}=0$ are obtained by solving the set of equations $(9)-(11)$. The solutions of the edge similarity equations (6) are applied as initial condition at $\xi=0$, and an implicit backward differencing in $\xi$ derivatives can be used. But the radial velocity $(F)$ changes sign within the boundary layer for $\xi>0.5$ and $n \leq 0$ which makes the coefficient of $F_{\xi}$, i.e., $-\xi(1-\xi) F$ as negative and hence a forward marching procedure in $\xi$ will lead to numerical instability. In those cases, in which the radial velocity may become positive, we have treated the partial differential equations (2) - (4) as a boundary value problem in which the boundary conditions in both $\xi$ and $\eta$ are prescribed. The solutions of the equations (6) and (7), respectively, governs the conditions at $\xi=0$ and $\xi=1$ and boundary conditions over $\eta$ are given by (5). A second-order accurate upwind difference scheme has been used for discretising the derivatives with respect to $\xi$ and a 
central difference has been used for $\eta$ derivatives. The Newton's linearisation technique has been used to cope with the non-linearity. The resulting system of linear algebraic equations has been solved by the block elimination method.

The equations (9)-(11) are discretised in the same way as has been described for discretisation of equations (2)-(4) with respect to $\xi$ and $\eta$ derivatives. The corresponding boundary conditions in $\xi$ are governed by the similarity equations which can be obtained by setting $\varphi=1, \partial / \partial t^{*}=0$ in equations (6) and (7).

For potential vortex flow $(n=1)$, no flow reversal in the boundary layer exists. In particular, numerical solutions for $n>0$ can be obtained upto a certain radial distance $\xi\left(0 \leq \xi \leq \xi_{0}<1\right)$ where the radial flow reversal does not take place. Hence the equations (2)-(4) can be solved by a forward integration in $\xi$ starting from $\xi=0$ (edge of the disk) by applying a backward difference approximation in $\xi$ derivatives.

The non-existence of the similarity solution near the center $(\xi=1)$ for the flow due to potential vortex $(n=1)$ can be proved mathematically in the following manner:

Integrating the equation (12a) twice with respect to $\eta$ from $\eta=0$ to $\eta=\eta_{e}\left(\eta_{e}\right.$ is the edge of the boundary layer) and using the boundary conditions (5) we get

$$
\frac{3-n}{4} f^{2}\left(\eta_{e}\right)=-\frac{3}{2}(1-n) \int_{0}^{\eta_{e}} \mathrm{~d} \eta \int_{\eta}^{\eta_{e}} F^{2} \mathrm{~d} \eta-\int_{0}^{\eta_{e}} \mathrm{~d} \eta \int_{\eta}^{\eta_{e}}\left(\theta-g^{2}\right) \mathrm{d} \eta .
$$

From (13) it is evident that for $f\left(\eta_{e}\right)$ to be real (i.e., for a physically meaningful solution to exist), it is necessary that $g^{2}$ should exceed $\theta$ at some point in the boundary layer, i.e. $g^{2}>\theta$ for some $\eta$ in $\left(0, \eta_{e}\right)$. For the sake of simplicity, we take $N=\operatorname{Pr}=1$ and by integrating the equations (12b) and (12c) twice with respect to $\eta$ and using the boundary conditions (5) we get for $n=1$

$$
\theta(\eta)=g(\eta)+\theta_{w}(1-g(\eta))
$$

From $(14), \theta(\eta) \geq g(\eta)$ for all $\eta$ in $0 \leq \eta \leq \eta_{e}$. Again,

$$
g_{\eta}=C \exp \left(-\int f \mathrm{~d} \eta\right)
$$

where $C$ is a constant of integration. Hence $g_{\eta}$ retains the same sign in $\left(0, \eta_{e}\right)$. So, $g(\eta)$ is a monotonic function varying from 0 to 1 . Thus, $g(\eta) \geq g^{2}(\eta)$ for all $\eta$. Hence we can conclude that

$$
\theta(\eta) \geq g(\eta) \geq g^{2}(\eta), \quad \text { for all } \eta \text { in } 0 \leq \eta \leq \eta_{e} .
$$

But (13) shows that for a physically meaningful solution to exist, $g^{2}(\eta)>\theta(\eta)$ for some $\eta$ which contradicts (16). So, we can conclude that for $n=1$ (potential vortex) the solution of (12) does not exist.

\section{Results and discussion}

The numerical solutions are obtained for Prandtl number $\operatorname{Pr}=0.72$ and $\omega=0.7$. The forms of $\varphi\left(t^{*}\right)$ considered are

$$
\varphi\left(t^{*}\right)=1+\varepsilon t^{* 2}, \varepsilon>0 ; \quad \varphi\left(t^{*}\right)=\left(1+\varepsilon_{1} \cos \left(\omega t^{*}\right)\right) /\left(1+\varepsilon_{1}\right)
$$

which corresponds to accelerated and oscillatory free stream flows respectively.

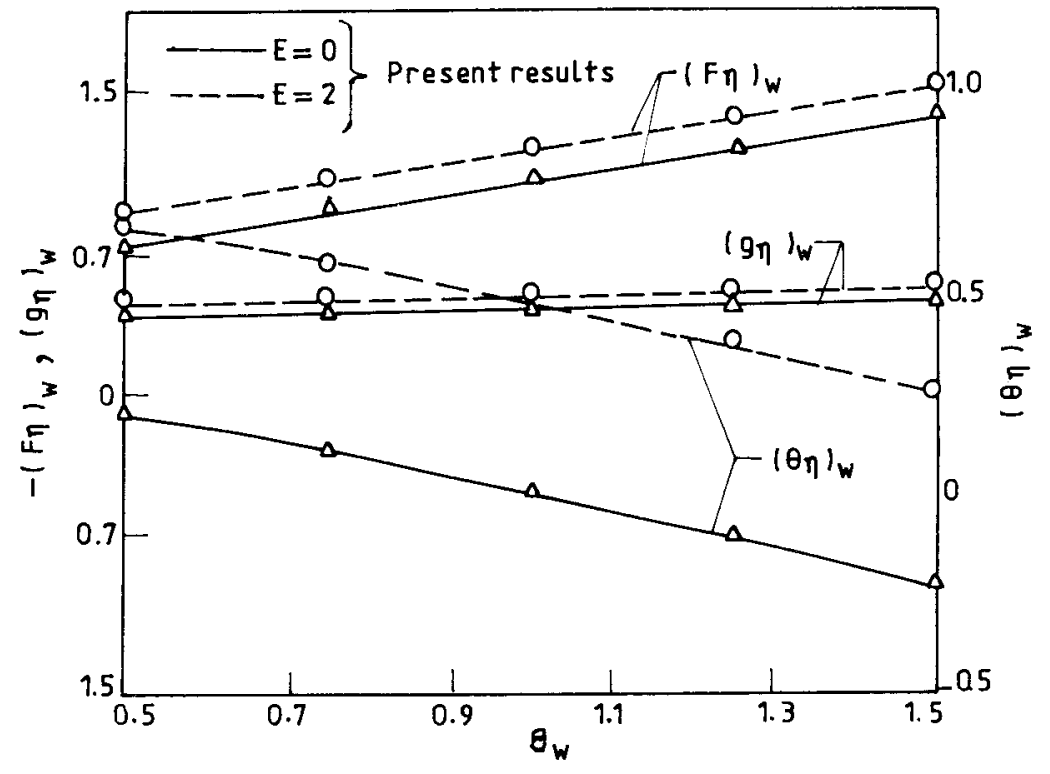

Fig. 1. Comparison of $\left(F_{\eta}\right)_{w},\left(g_{\eta}\right)_{w}$, $\left(\theta_{\eta}\right)_{w}$ at the edge $(\xi=0)$ with ToREN et al. [9] for $n=-1, \operatorname{Pr}=0.7$, $\omega=1.0,(\Delta) E_{1}=0 ;(O) E_{1}=2$, ToREN et al. [9] 
Table 1. Comparison of $\left(F_{\eta}\right)_{w},\left(g_{\eta}\right)_{w}$ for incompressible case as a function of $n$ when $t^{*}=0$

\begin{tabular}{|c|c|c|c|c|c|c|}
\hline$\xi$ & Flow Parameters & $\begin{array}{l}n=-1 \\
\text { present results }\end{array}$ & $\begin{array}{l}n=-1 \\
\text { Van de Vooren } \\
\text { et al. results }\end{array}$ & $\begin{array}{l}n=0 \\
\text { present results }\end{array}$ & $\begin{array}{l}n=0.5 \\
\text { present results }\end{array}$ & $\begin{array}{l}n=1 \\
\text { present results }\end{array}$ \\
\hline \multirow[t]{2}{*}{0} & $-\left(F_{\eta}\right)_{w}$ & $\begin{array}{l}1.0686 \\
(1.0738)\end{array}$ & 1.0681 & $\begin{array}{c}1.0680 \\
(1.0738)\end{array}$ & $\begin{array}{c}1.0682 \\
(1.0738)\end{array}$ & $\begin{array}{c}1.0682 \\
(1.0783)\end{array}$ \\
\hline & $\left(g_{\eta}\right)_{w}$ & $\begin{array}{c}0.4392 \\
(0.4200)\end{array}$ & 0.4396 & $\begin{array}{c}0.4396 \\
(0.4200)\end{array}$ & $\begin{array}{c}0.4394 \\
(0.4200)\end{array}$ & $\begin{array}{c}0.4394 \\
(0.4200)\end{array}$ \\
\hline \multirow[t]{2}{*}{0.865} & $-\left(F_{\eta}\right)_{w}$ & $\begin{array}{c}0.9822 \\
(0.9702)\end{array}$ & 0.9704 & $\begin{array}{l}1.1645 \\
(1.1533)\end{array}$ & $\begin{array}{l}1.1640 \\
(1.1433)\end{array}$ & $\begin{array}{l}1.1302 \\
(1.0881)\end{array}$ \\
\hline & $\left(g_{\eta}\right)_{w}$ & $\begin{array}{c}0.7477 \\
(0.7474)\end{array}$ & 0.7469 & $\begin{array}{c}0.4581 \\
(0.4617)\end{array}$ & $\begin{array}{c}0.2506 \\
(0.2707)\end{array}$ & $\begin{array}{c}0.1307 \\
(0.1453)\end{array}$ \\
\hline \multirow[t]{2}{*}{0.777} & $-\left(F_{\eta}\right)_{w}$ & $\begin{array}{c}1.0180 \\
(1.0161)\end{array}$ & 1.0123 & $\begin{array}{c}1.1510 \\
(1.1466)\end{array}$ & $\begin{array}{c}1.1390 \\
(1.1323)\end{array}$ & $\begin{array}{c}1.1050 \\
(1.0920)\end{array}$ \\
\hline & $\left(g_{\eta}\right)_{w}$ & $\begin{array}{c}0.7256 \\
(0.7388)\end{array}$ & 0.7266 & $\begin{array}{c}0.4563 \\
(0.4583)\end{array}$ & $\begin{array}{c}0.3001 \\
(0.3087)\end{array}$ & $\begin{array}{c}0.1858 \\
(0.1963)\end{array}$ \\
\hline \multirow[t]{2}{*}{0.393} & $-\left(F_{\eta}\right)_{w}$ & $\begin{array}{c}1.1040 \\
(1.1053)\end{array}$ & 1.1131 & $\begin{array}{c}1.1121 \\
(1.1059)\end{array}$ & $\begin{array}{c}1.1039 \\
(1.0973)\end{array}$ & $\begin{array}{c}1.0932 \\
(1.0885)\end{array}$ \\
\hline & $\left(g_{\eta}\right)_{w}$ & $\begin{array}{c}0.5605 \\
(0.5608)\end{array}$ & 0.5577 & $\begin{array}{c}0.4446 \\
(0.4459)\end{array}$ & $\begin{array}{c}0.3912 \\
(0.3936)\end{array}$ & $\begin{array}{c}0.3432 \\
(0.3423)\end{array}$ \\
\hline 1.000 & $\begin{array}{l}-\left(F_{\eta}\right)_{w} \\
\left(g_{\eta}\right)_{w}\end{array}$ & $\begin{array}{c}0.9422 \\
(0.9420) \\
0.7722 \\
(0.7730)\end{array}$ & 0.7727 & $\begin{array}{c}1.1988 \\
(1.2010) \\
0.4442 \\
(0.4430)\end{array}$ & & \\
\hline
\end{tabular}

Results in parentheses are those of BELCHER et al. [4]

In order to assess the accuracy of our method, we have compared our result for the corresponding steady $\left(t^{*}=0\right)$ incompressible case $(\theta=1)$ with those of BELCHER et al. [4] for various values of $n(-1 \leq n \leq 1)$ and with those of VAN DE VoOREn et al. [6] for $n=-1$ (see Table 1). Comparisons are also made with the compressible edge similarity solutions of TOREN and SOLAn [10] (see Fig. 1).

The effect of Mach number on skin friction parameters $\left(\left(F_{\eta}\right)_{w},\left(g_{\eta}\right)_{w}\right)$, and heat transfer parameter $\left(\theta_{\eta}\right)_{w}$ for the steady case $\left(t^{*}=0\right)$ and $\theta_{w}=0.7$ is illustrated in Fig. 2. With the increase of Mach number the magnitude of radial skin friction $\left(F_{\eta}\right)_{w}$ and the heat transfer $\left(\theta_{\eta}\right)_{w}$ increases. These effects are more pronounced near the edge of the disk. But the tangential skin friction $\left(g_{\eta}\right)_{w}$ is little affected by the variation of $M$.

Here we have presented all the results for Mach number $M=1$. In Fig. 3 the radial and tangential velocity profiles are shown for different values of the vortex parameter $n(-1 \leq n \leq 1)$ at $\xi=0.4$ when $\theta_{w}=0.7$ (cold disk) and $t^{*}=2$ in an accelerating free stream. The velocity profiles approach their asymptotic values monotonically over the outer half of the disk. Increase of $n$ increases the radial inflow $-F$.

Fig. 4 shows the radial $(F)$ and tangential velocity $(g)$ profiles at time $t^{*}=2$ in an accelerating free stream $\left(\varphi\left(t^{*}\right)=1+0.2 t^{* 2}\right)$ which is in rigid-body rotation over a hot disk $\left(\theta_{w}=1.3\right)$ at various values of $\xi$ when Mach number $M=1$. Both the radial and tangential velocity profiles exhibit oscillation about their asymptotic values as $\xi$ increases. We find here that the boundary layer at the edge of the disk begins by acting as a centripetal fan drawing

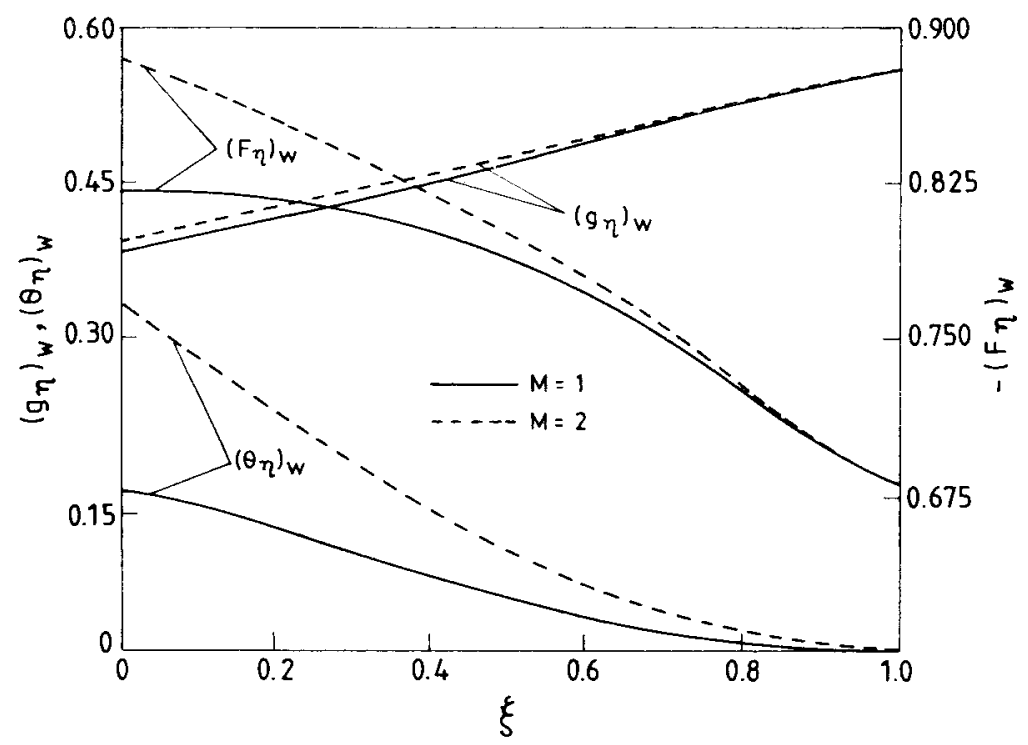

Fig. 2. Effect of Mach number $(M)$ on $\left(F_{\eta}\right)_{w},\left(g_{\eta}\right)_{w}$, and $\left(\theta_{\eta}\right)_{w}$ for $t^{*}=0$, $\theta_{w}=0.7, n=-1, \quad \operatorname{Pr}=0.7, \omega=0.7$, $M=1,2$ 


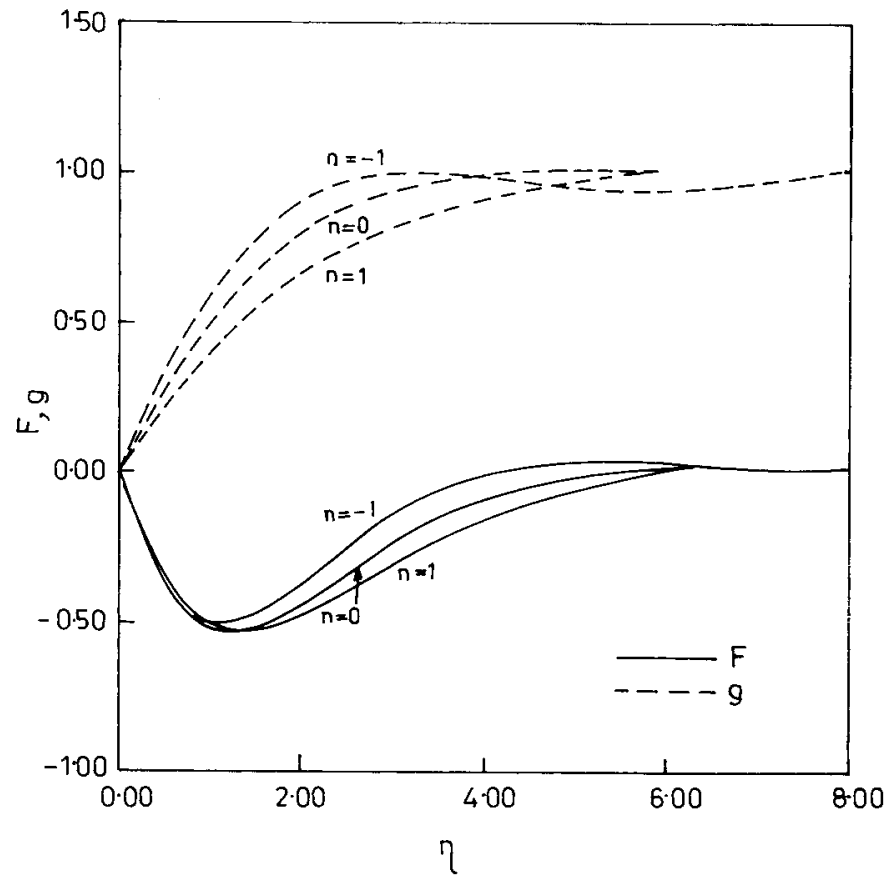

Fig. 3. Profiles of radial and tangential velocity at time $t^{*}=2$ in accelerating free stream when $\xi=0.4, \quad \theta_{w}=0.7, \quad n=-1,0,1, \quad \operatorname{Pr}=0.7$, $\omega=0.7, M=1$

fluid radially inwards and throwing it axially outwards. But over the inner half of the disk $(\xi \geq 0.5)$ radial flow reversal takes place and the boundary layer flow gradually approaches the terminal similarity of Bödewadt's type. BELCHER et al. [4] have also observed that for $n<0$ the circulation of the outer flow is an increasing function of radius which causes the radial and tangential velocity profiles in the boundary layer to oscillate about their inviscid values for any $\xi>0$ with amplitude that diminish exponentially as the outer edge of the boundary layer is approached.

The boundary layer over the inner half of the disk depends essentially on the inviscid flow field and has completely forgotten the manner in which it originates at the edge of the disk. But the potential vortex yields a boundary layer which cannot forget its history. It starts at the outer edge of the disk and continues to take inflow until it erupts. We have shown that for potential vortex flow $(n=1)$ the boundary layer does not exist near the center $(\xi=1)$. It has been found numerically that for all $n$ in $-1 \leq n \leq 1$ solutions exist only upto $\xi=0.4$ for cold disk $\left(\theta_{w}=0.7\right)$ and it is upto $\xi=0.2$ for the flow over a hot disk $\left(\theta_{w}=1.3\right)$.

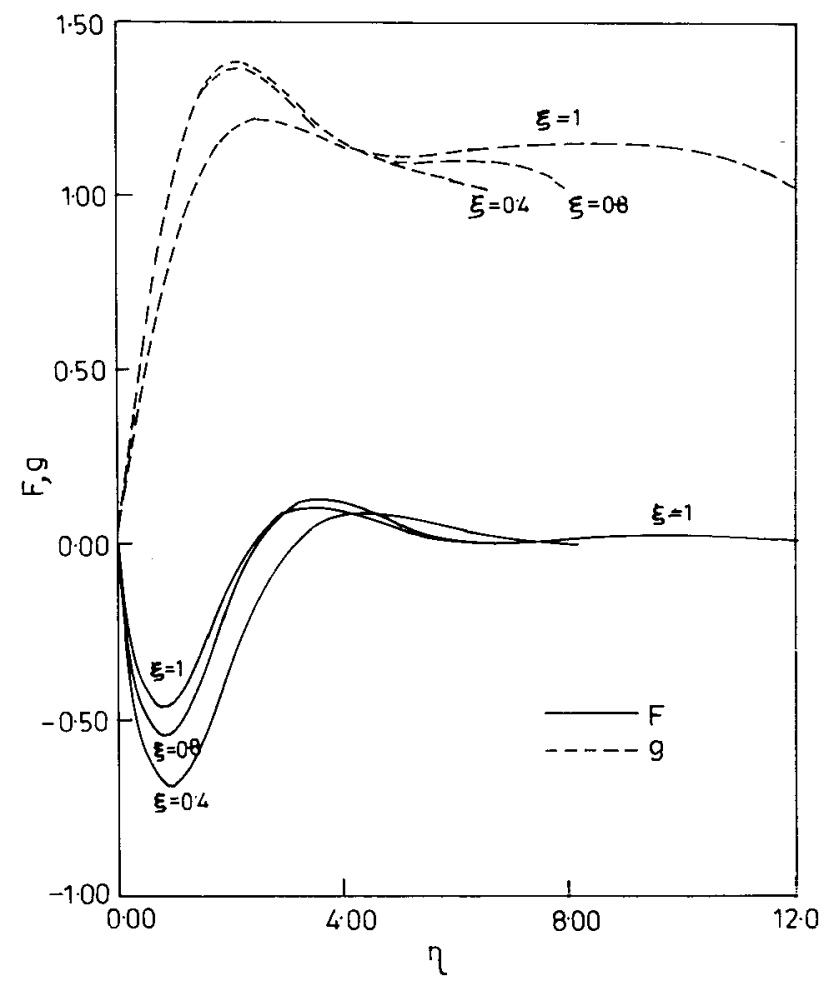

Fig. 4. Profiles of radial and tangential velocity at time $t^{*}=2$ in accelerating free stream when $\xi=1.0,0.4,0.8, \theta_{w}=1.3, n=-1, \operatorname{Pr}=0.7, \omega=0.7$, $M=1$ 

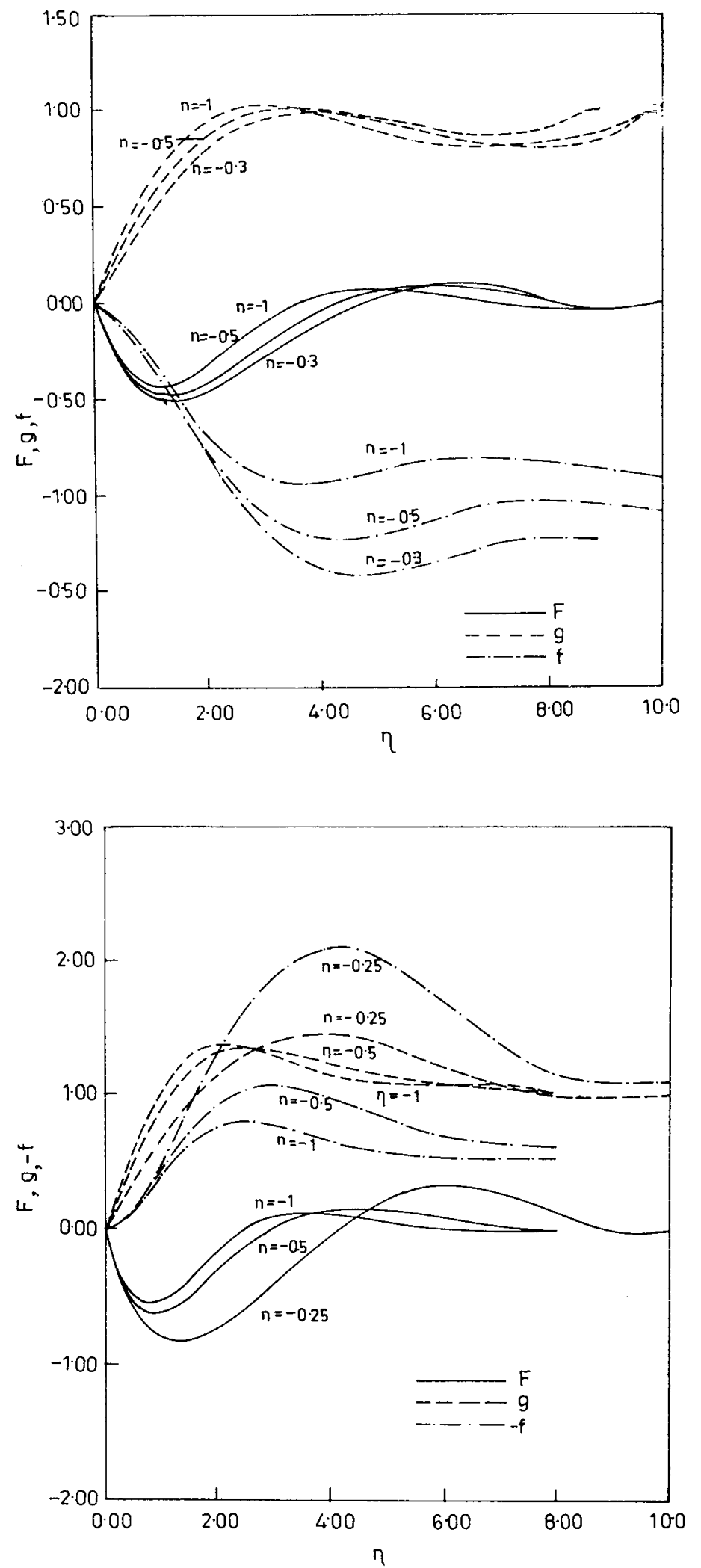

Fig. 5. Profiles of radial velocity, tangential velocity, and axial velocity at time $t^{*}=2$ in accelerating free stream when $\xi=0.8$, $\theta_{w}=0.7, \quad n=-1,-0.5,-0.3, \quad \operatorname{Pr}=0.7$, $\omega=0.7, M=1$

Fig. 6. Profiles of radial velocity, tangential velocity, and axial velocity at time $t^{*}=2$ in accelerating free stream when $\xi=0.8$, $\theta_{w}=1.3, \quad n=-1,-0.5,-0.25, \quad \operatorname{Pr}=0.7$, $\omega=0.7, M=1$

The numerical solutions for the entire range of the radial distance $0 \leq \xi \leq 1$ cannot be obtained beyond certain critical value $n_{0}$ of the vortex parameter $n,-1 \leq n \leq n_{0}$. We have seen that the critical value of $n$, i.e., $n_{0}=0.3$ for $\theta_{w}=0.7$ (cold disk) and $n_{0}=-0.25$ for $\theta_{w}=1.3$ (hot disk).

Figs. 5 and 6 illustrate the effect of the vortex parameter $n,-1 \leq n \leq n_{0}$, on the velocity profiles $(f, F, g)$ at $\xi=0.8$ at the time $t^{*}=2$ in an accelerating free stream $\left(\varphi\left(t^{*}\right)=1+0.2 t^{* 2}\right)$ when $\theta_{w}=0.7$ and 1.3 . The radial outflow and the overshoot in tangential velocity are taking place. Increase of $n$ produces an increment in the radial inflow or outflow but reduces the tangential velocity near the disk. Axial velocity $(-f)$ increases with the increase of $n$. It is 

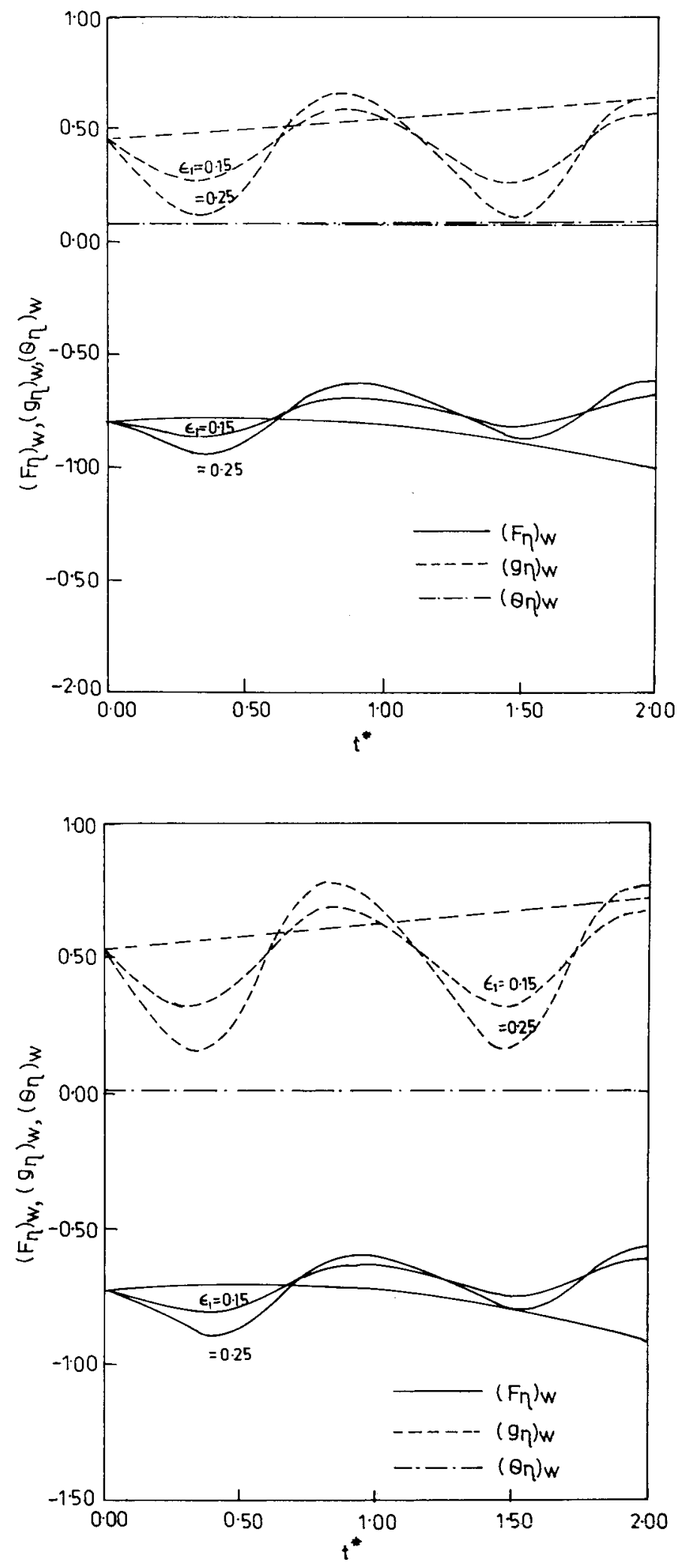

Fig. 7a. Variation of $\left(F_{\eta}\right)_{w},\left(g_{\eta}\right)_{w},\left(\theta_{\eta}\right)_{w}$ with $t^{*}$ when $\xi=0.4, \theta_{w}=0.7, n=-1, \operatorname{Pr}=0.7$, $\omega=0.7, \varepsilon_{1}=0.15,0.25, M=1$ in both accelerating and oscillating free stream
Fig. 7b. Variation of $\left(F_{\eta}\right)_{w},\left(g_{\eta}\right)_{w},\left(\theta_{\eta}\right)_{w}$ with $t^{*}$ when $\xi=0.8, \theta_{w}=0.7, n=-1, \operatorname{Pr}=0.7$, $\omega=0.7, \varepsilon_{1}=0.15,0.25, M=1$ in both accelerating and oscillating free stream

evident from Figs. 5-6 that the amplitude of oscillation in velocity profiles $(F, g)$ increases with the increase of the vortex parameter $n$. Rise in disk temperature $\left(\theta_{w}\right)$ produces an increment in the magnitude of the velocities.

Effect of the non-dimensional time $t^{*}\left(0 \leq t^{*} \leq 2\right)$ on radial skin friction $\left(F_{\eta}\right)_{w}$, tangential skin friction $\left(g_{\eta}\right)_{w}$, and heat transfer $\left(\theta_{\eta}\right)_{w}$ are shown in Figs. $7 \mathrm{a}$ and $7 \mathrm{~b}$. A rigid rotation $(n=-1)$ of the accelerating free stream $\left(\varphi\left(t^{*}\right)=1+0.2 t^{* 2}\right)$ and oscillating free stream $\left(\varphi\left(t^{*}\right)=1+\varepsilon_{1} \cos \left(5.6 t^{*}\right) /\left(1+\varepsilon_{1}\right)\right)$ over a cold disk $\left(\theta_{w}=0.7\right)$ are considered. Graphs are depicted for two different radial distances $\xi(=0.4,0.8)$. In an accelerating free stream, the magnitude of both $\left(F_{\eta}\right)_{w}$ and $\left(g_{\eta}\right)_{w}$ increases with the increase of time and they oscillate in an oscillating free stream. 

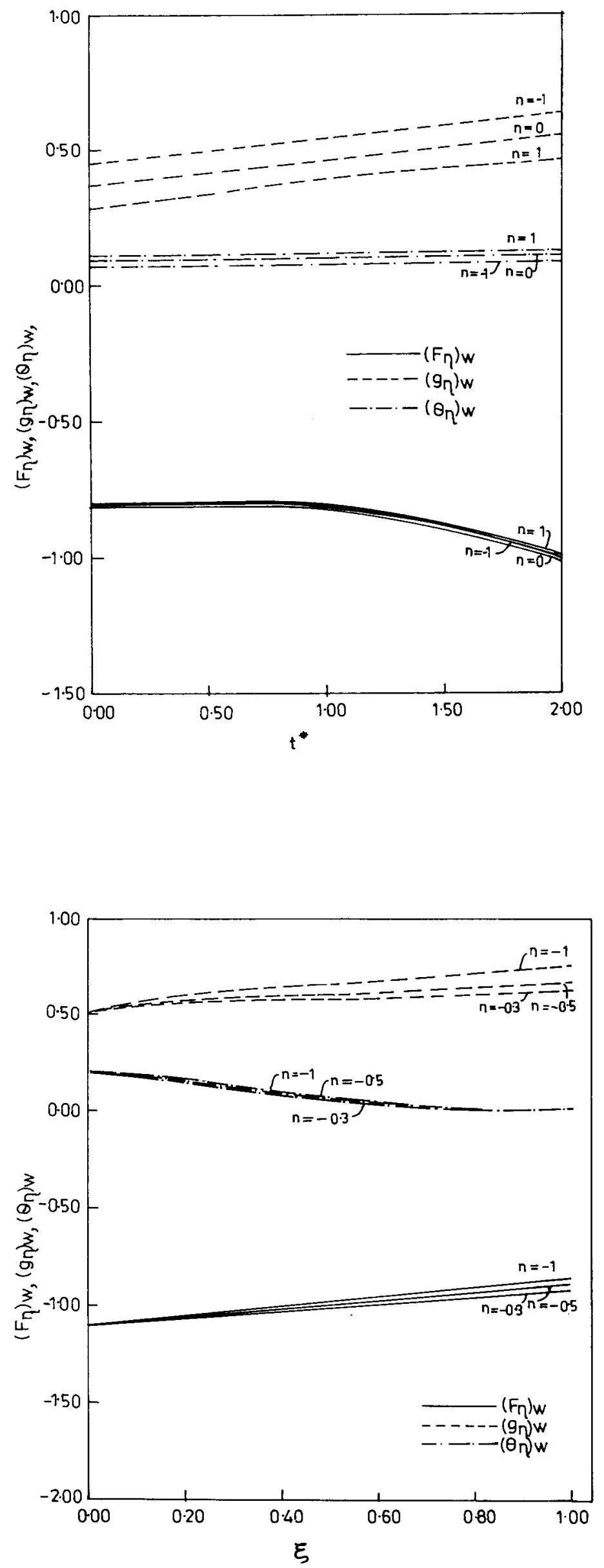

Fig. 8. Variation of $\left(F_{\eta}\right)_{w},\left(g_{\eta}\right)_{w},\left(\theta_{\eta}\right)_{w}$ with $t^{*}$ when $\xi=0.4, \theta_{w}=0.7, n=-1,0,1, \operatorname{Pr}=0.7$, $\omega=0.7, M=1$ in accelerating free stream
Fig. 9. Variation of $\left(F_{\eta}\right)_{w},\left(g_{\eta}\right)_{w},\left(\theta_{\eta}\right)_{w}$ at time $t^{*}=2$ with $\xi$ when $\theta_{w}=0.7$, $n=-1.0,-0.5,-0.3, \quad \operatorname{Pr}=0.7, \quad \omega=0.7$, $M=1 \mathrm{in}$ accelerating free stream 
The amplitude of oscillation in $\left(F_{\eta}\right)_{w}$ and $\left(g_{\eta}\right)_{w}$ increases with the increase of $\varepsilon_{1}$. It is evident from the Figs. $7 \mathrm{a}$ and $7 \mathrm{~b}$ that the tangential skin friction $\left(g_{\eta}\right)_{w}$ responds more to the fluctuation in the free stream angular velocity than the radial skin friction $\left(F_{\eta}\right)_{w}$.

Fig. 8 describes the effect of the vortex parameter $n$ in $[-1,1]$ on the radial and tangential skin friction and heat transfer parameter at different time $t^{*}, 0 \leq t^{*} \leq 2$, in an accelerating free stream $\left(\varphi\left(t^{*}\right)=1+0.2 t^{* 2}\right)$ at a radial distance $\xi=0.4$ with $\theta_{w}=0.7$ (cold disk). Increase in $n$ produces as reduction in the tangential skin friction $\left(g_{\eta}\right)_{w}$. Magnitude of the radial skin friction is least for potential vortex flow for $0 \leq t^{*} \leq 2$. Heat transfer has relatively little effect due to the variation of $n$.

The variation of radial skin friction, tangential skin friction, and heat transfer with the radial distance $\xi, 0 \leq \xi \leq 1$, has been illustrated in Fig. 9. Graphs are depicted at a non-dimensional time $t^{*}=2$ in an accelerating free stream $\left(\varphi\left(t^{*}\right)=1+0.2 t^{* 2}\right)$ for various values of the vortex parameter $n(=-1,-0.5,-0.3)$ when $\theta_{w}=0.7$ (cold disk). The tangential skin friction $\left(g_{\eta}\right)_{w}$ increases as $\xi$ increases but the magnitude of radial skin friction $\left(F_{\eta}\right)_{w}$ and heat transfer $\left(\theta_{\eta}\right)_{w}$ get reduced with the increase of $\xi$. Increase of $n$ in $[-1,-0.3]$ gives rise to an increment in the magnitude of radial skin friction but reduces the tangential skin friction.

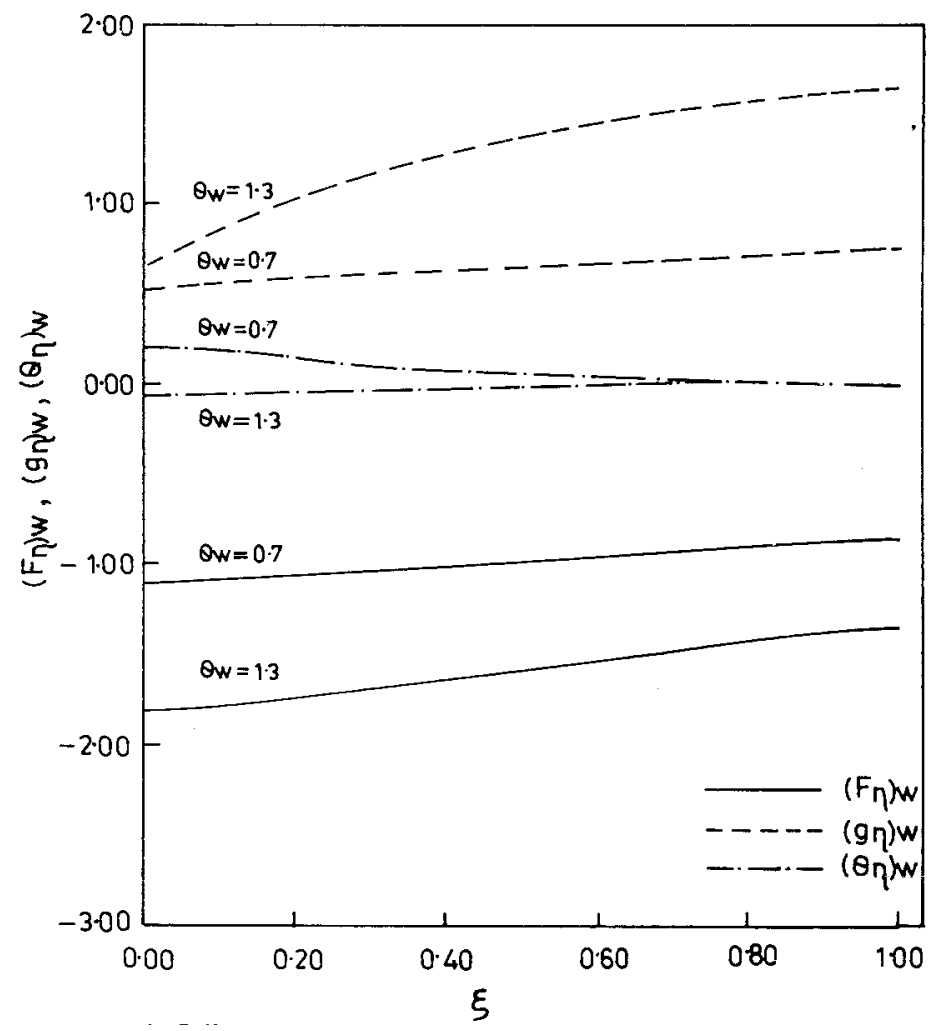

Fig. 10. Variation of $\left(F_{\eta}\right)_{w},\left(g_{\eta}\right)_{w},\left(\theta_{\eta}\right)_{w}$ at time $t^{*}=2$ with $\xi$ when $\theta_{w}=0.7,1.3$, $n=-1, \operatorname{Pr}=0.7, \omega=0.7, M=1$ in accelerating free stream

Fig. 10 shows the effect of disk temperature $\left(\theta_{w}=1.3,0.7\right)$ on radial and tangential skin friction and heat transfer for the entire range of the radial distance $\xi, 0 \leq \xi \leq 1$, at time $t^{*}=2$ in an accelerating free stream. The free stream considered to be in rigid-body rotation $(n=-1)$. Rise in disk temperature produces an increment in the magnitude of radial and tangential skin friction but reduces the magnitude of heat transfer. Effect of temperature is more pronounced on tangential skin friction $\left(g_{\eta}\right)_{w}$ than the radial skin friction $\left(F_{\eta}\right)_{w}$.

\section{Conclusions}

The boundary layer over a finite disk of a compressible fluid which begins at the edge of the disk and grows inward toward the center is studied numerically. Velocity profiles near the edge of the disk are found to be non-oscillatory but from the mid-radius and onwards radial and tangential velocities (for $n<1$ ) exhibit oscillation about their free stream values and approach their terminal similarity profiles of Bödewadt's type. A solution exists for a certain radial distance $(\xi>0)$ for the entire range of $n$ in $[-1,1]$ and for a certain range of $n<0$ for entire range of $\xi, 0 \leq \xi \leq 1$. The nonexistence of solution of the terminal similarity equations for $n=1$ is also proved. Magnitude of skin friction parameters increases with time in an accelerating free stream and they exhibit oscillation in an oscillating free stream. Skin friction parameters increase with the rise of disk temperature. 


\section{References}

1 Karman, T. von: Über laminare und turbulente Reibung. Z. Angew. Math. Mech. 1 (1921), 233-252.

2 Stewartson, K.: Boundary layer research. Symp., Freiburg, Springer 1958.

3 Rogers, M. H.; Lance, G. N.: The boundary layer on a disc of finite radius in a rotating fluid. Q. J. Mech. Appl. Math. 17 (1964), 319-330.

4 Belcher, R. J.; Burggraf, O. R.; Stewartson, K.: On generalised vortex boundary layers. J. Fluid Mech. 52 (1972), $753-780$.

5 Bodonyi, R. J.; Stewartson, L.: Boundary layer similarity near the edge of a rotating disk. ASME J. Appl. Mech. 42 (1975), $584-590$.

6 Van de Vooren, A. I.; Botta, E. F. F.; Stout, J.: The boundary layer on a disk at rest in a rotating fluid. Q. J. Mech. Appl. Math. 40 (1987), 15-33.

7 VAn de Vooren, A. I.: The connection between Ekman and Stewartson layers for a rotating disk. J. Engng. Math. 27 (1993), 189-207.

8 Brady, J. F.; Durlofsky, L.: On rotating disk flow. J. Fluid Mech. 175 (1987), 363-394.

9 Toren, M.; Solan, A.: Laminar boundary layer on a finite disk in a rotating compressible isothermal flow. ASME J. Fluids Engng. 101 (1979), 166-172.

10 Toren, M.; Solan, A.: Rotating compressible flow ower the edge of a finite disk. ASME J. Appl. Mech. 48 (1981), 249-254.

11 King, W. S.; Lewellen, W. S.: Boundary layer similarity solutions for rotating flow with and without magnetic interaction. Phys. Fluids 7 (1964), 1674-1680.

12 Burgaraf, O. R.; Stewartson, K.; Belcher, J.: Boundary layer induced by a potential vortex. Phys. Fluids. 14 (1971), $1821-1833$.

13 Telionis, D. P.: Review unsteady boundary layers. ASME J. Fluids Engng. 101 (1979), 29-43.

14 Bödewadt, U. T.: Die Drehströmung über festem Grunde. Z. Angew. Math. Mech. 20 (1940), 241-253.

15 Riley, N.: Thermally induced boundary layer flows in a rotating environment. J. Fluid Mech. 29 (1967), 241-257.

16 Bhattacharyya, S.; NAth, G.: Unsteady swirling flow of a compressible fluid over an infinite disk with a magnetic field or suction. Int. J. Non-linear Mech. 27 (1992), 391-404.

Received July 15, 1996, revised and accepted version May 6, 1997

Addresses: Dr. S. Bhattacharyya, Dr. A. Pal, Dr. N. Datta, Department of Mathematics, Indian Institute of Technology, Kharagpur-721 302, India; Dr. G. NATH, Department of Mathematics, Indian Institute of Science, Bangalore-560 012, India 